\title{
Regulation of the Hypothalamic-Pituitary-Adrenocortical System in Patients with Panic Disorder
}

\author{
Angelika Erhardt', Marcus Ising', Paul Gerson Unschuld', Nikola Kern',2, Susanne Lucae', Benno Pütz', \\ Manfred Uhr', Elisabeth B Binder',3, Florian Holsboer' and Martin E Keck*,' \\ 'Max Planck Institute of Psychiatry, Munich, Germany
}

\begin{abstract}
Anxiety and depressive disorders are among the most common psychiatric disorders with a high number of hospital admissions and a lifetime prevalence of up to $25 \%$. So far, the pathophysiological mechanisms for anxiety disorders remain to be found. Preclinical studies suggest that changes in hypothalamic-pituitary-adrenocortical (HPA) system function are causally related to the expression of anxietyrelated behavior. The findings on HPA system function in patients with anxiety disorders are, however, heterogeneous. Both hypo- and hyperresponsiveness of HPA response in various anxiety disorders under different experimental conditions were found. In order to characterize putative case/control differences in HPA system function, we performed a Dex-CRH test, a widely used test to pick up changes in HPA system regulation with high sensitivity, in 30 patients with panic disorder, 35 patients with major depressive episode and in 30 controls individually matched for ethnicity, age and gender. The results indicate a similar dysregulation of the HPA system response in the Dex-CRH test in both patient groups. This finding further underlines the hypothesis that both, depression and panic disorder, share impaired HPA system regulation, supporting the notion that the impairment is involved in the pathophysiology of these clinical conditions. However, differences in the suppression effects and psychopathological correlation patterns between depressed and panic patients suggest different biological mechanisms of HPA system dysregulation in both disorders.

Neuropsychopharmacology (2006) 3 I, 25 I5-2522. doi:I0.1038/sj.npp. I 30 I I68; published online 12 July 2006
\end{abstract}

Keywords: panic disorder; anxiety; major depression; combined dexamethasone corticotropin-releasing hormone challenge test (Dex-CRH test); ACTH; cortisol

\section{INTRODUCTION}

Panic disorder is a highly prevalent (5\%) clinical condition causing a high number of hospital admissions (Merikangas et al, 1996; Roy-Byrne et al, 2000). Although the exact pathophysiological mechanisms underlying panic disorder have not been identified yet, distinct biological hypothesis have been posited and preclinical and clinical studies point towards the importance of stress hormone regulation as one pathological condition in anxiety (eg Schreiber et al, 1996; Condren et al, 2002; review: Landgraf and Wigger (2002)).

Profound changes in hypothalamic-pituitary-adrenocortical (HPA) system regulation have been demonstrated in major depression, and the dexamethasone-suppression/

\footnotetext{
* Correspondence: Dr ME Keck. Current address: Division of Psychiatry Research, Psychiatric University Hospital, CH-8032 Zurich, Switzerland, Tel: +4| 44389 |477, Fax: + 4| 44389 |4I4,

E-mail: martin.keck@puk.zh.ch

${ }_{3}^{2}$ Current address: King's College, Maudsley Hospital, London, UK ${ }^{3}$ Current address: Emory University School of Medicine, Atlanta, GA 30322, USA

Received 24 March 2006; revised I June 2006; accepted I 3 June 2006 Online publication: 16 June 2006 at http://www.acnp.org/citations/ Npp061606060195/default.pdf
}

corticotropin-releasing hormone-stimulation (Dex-CRH) test has proven to be a sensitive tool for detecting such altered HPA regulation (Heuser et al, 1994; Holsboer, 2000; Künzel et al, 2003; Ising et al, 2005). One of the major modulators of the HPA system is corticotropin releasing hormone (CRH) (review: Engelmann et al (2004); Nemeroff and Vale (2005)), which elicits the release of pituitary ACTH and subsequently cortisol under basal conditions and in response to stress. In rodents, the intracerebroventricular injections of CRH trigger an anxiogenic response (Carrasco and Van de Kar, 2003), whereas antagonism of the CRH receptor 1 under specific experimental conditions and the conditional inactivation of CRH receptor 1 in limbic brain regions results in anxiolysis in rats and mice (eg Keck et al, 2001; van Gaalen et al, 2002; Müller et al, 2003) and also anxiety scores were reported to decrease under treatment of depressives with a CRHR1 antagonist (Zobel et al, 2000). These findings suggest that $\mathrm{CRH}$ as a neuropeptide has an important modulatory function on anxiety-related behavior (review: Keck et al (2005)). Anxiety is a common core symptom in depressed patients and there is a high comorbidity rate between anxiety and depressive disorders (Gorman and Coplan (1996); Roy-Byrne et al (2000); Hettema et al (2001)). Moreover, anxiety disorders seem 
to precede the development of depression (Goodwin et al (2004)) suggesting that there might be a continuum between these disorders with common pathophysiological features. As both disorders imply inappropriate adaptation to stressors they are viewed as stress-related disorders suggesting a causal role of HPA system dysregulation (review: De Kloet et al (2005)). So far, however, the findings on HPA system function in patients suffering from anxiety disorders have led to inconclusive results. The majority of studies suggest that basal plasma cortisol and ACTH concentrations in patients with panic disorder are unaltered (Brambilla et al (1992); Targum (1992)). Some studies, however, reported elevations of basal plasma and salivary cortisol in panic patients (Goldstein et al, 1987; Wedekind et al, 2000; Bandelow et al, 2000b). In response to the pharmacological induction of panic attacks with the panicogen lactate, an increase in cortisol was observed during the anticipation of panic attacks but not during the attacks themselves (Coplan et al, 1998). Under the conditions of acute mild stress, significantly higher cortisol levels in panic disorder patients have been found compared to healthy controls (Stones et al, 1999), whereas the majority of studies have shown a normal suppression of ACTH and cortisol in the standard dexamethasone suppression test (DST) and Dex-CRH test (Goldstein et al, 1987; Okasha et al, 1994; Schreiber et al, 1996).

Taken together, there is some evidence for abnormalities in HPA system regulation in panic disorder. Given the anxiogenic properties of $\mathrm{CRH}$ mentioned above and the distribution of $\mathrm{CRH}$ receptors in brain areas known to be involved in the modulation of anxiety-related behavior, the hypothalamic CRH and related neurocircuits are likely to be involved in the pathogenesis of panic disorder (review: Gorman et al (2000)). In the present study, we applied the Dex-CRH test to further characterize the function of the HPA system in panic disorder compared to patients suffering from a major depressive disorder (MDD) and healthy controls. We also examined the effects of different psychopathological phenotypes upon Dex-CRH test induced hormone response. We used the Dex-CRH test in our study because this test was consistently shown to have high sensitivity in detecting even subtle HPA system dysregulations which are not necessarily reflected in differences in basal peripheral plasma ACTH and corticosteroid concentrations (Holsboer, 2000; Pariante et al, 2004; Ising et al, 2005; Künzel et al, 2003). This test is aimed at detecting impaired glucocorticoid feedback signaling at the pituitary and to evaluate the effects of an altered release of central neuropeptides involved in the regulation of the HPA system, such as CRH and vasopressin (AVP) (Keck and Holsboer, 2001). Moreover, the Dex-CRH test was shown to be rather refractory against disease-unrelated factors such as cigarette smoking, weight, age and test-concomitant acute stress and a current psychopharmacological treatment does not appear to affect test outcome in acutely ill patients (Künzel et al, 2003).

\section{MATERIALS AND METHODS}

\section{Subjects}

A total of 30 outpatients suffering from panic disorder from our anxiety disorder clinic were included. The second group consists of 35 in-patients admitted to our psychiatric hospital for treatment of a single or recurrent major depressive episode. In both groups, comorbid psychiatric disorders, such as specific phobias and personality disorders, were recorded but did not present an exclusion criterion (Table 1). Overall psychiatric comorbidity was observed in $6.7 \%$ of patients in the panic disorder group and in $5.7 \%$ in the MDD group. Patients with panic disorder and comorbid depression were not included in the study. Demographic data, psychiatric diagnoses and comorbidities are listed in Tables 1 and 2. Patients with panic disorder and/or depression due to a neurological or medical illness were excluded from the study.

The diagnoses were ascertained by trained psychiatrists according to DSM-IV and were confirmed with the Structured Clinical Interview for DSM-III-R (SCID; German version SKID; Wittchen et al, 1990). Disease and family histories were obtained by a direct interview and from medical records. The age of onset, the duration of pharmacological treatment and, in patients with MDD, the number of previous episodes and duration of the index episode were assessed (Table 2).

The severity of psychopathology at admission was assessed through several clinically validated scales. We used the Hamilton Anxiety (HAMA) (Hamilton, 1959) and Hamilton Depression (HAM-D) scales, the HAM-Ds factorial subscales 'vegetative depression' and 'cognitive depression' according to Overall and Rhoades (1982), State and Trait Anxiety Inventory scale (Spielberger, 1983), Panic and Agoraphobia scale (Bandelow et al, 2000a), including selfrating and ratings assessed by clinicians.

As control subjects 30 healthy volunteers with no personal or family history of psychiatric disorders were included. All participants underwent a thorough clinical interview (SCID I and II) and physical examination, including blood analysis, drug monitoring and cranial magnetic resonance imaging. Volunteers were not compensated for participation.

Table I Primary Diagnosis
PD $(n=30)$
MDD $(n=35)$

\begin{tabular}{|c|c|c|c|c|}
\hline \multirow[t]{2}{*}{ Diagnosis } & PD and agoraphobia (F40.0I) & $19(63.3 \%)$ & Single MDE (F32.2) & $9(25.7 \%)$ \\
\hline & $\mathrm{PD}(\mathrm{F} 4 \mathrm{I} .0)$ & II (36.7\%) & Recurrent unipolar MDD (F33.2) & $26(74.3 \%)$ \\
\hline
\end{tabular}

PD: panic disorder; MDE: major depressive episode. 
Table 2 Demographic Data and Clinical Characteristics

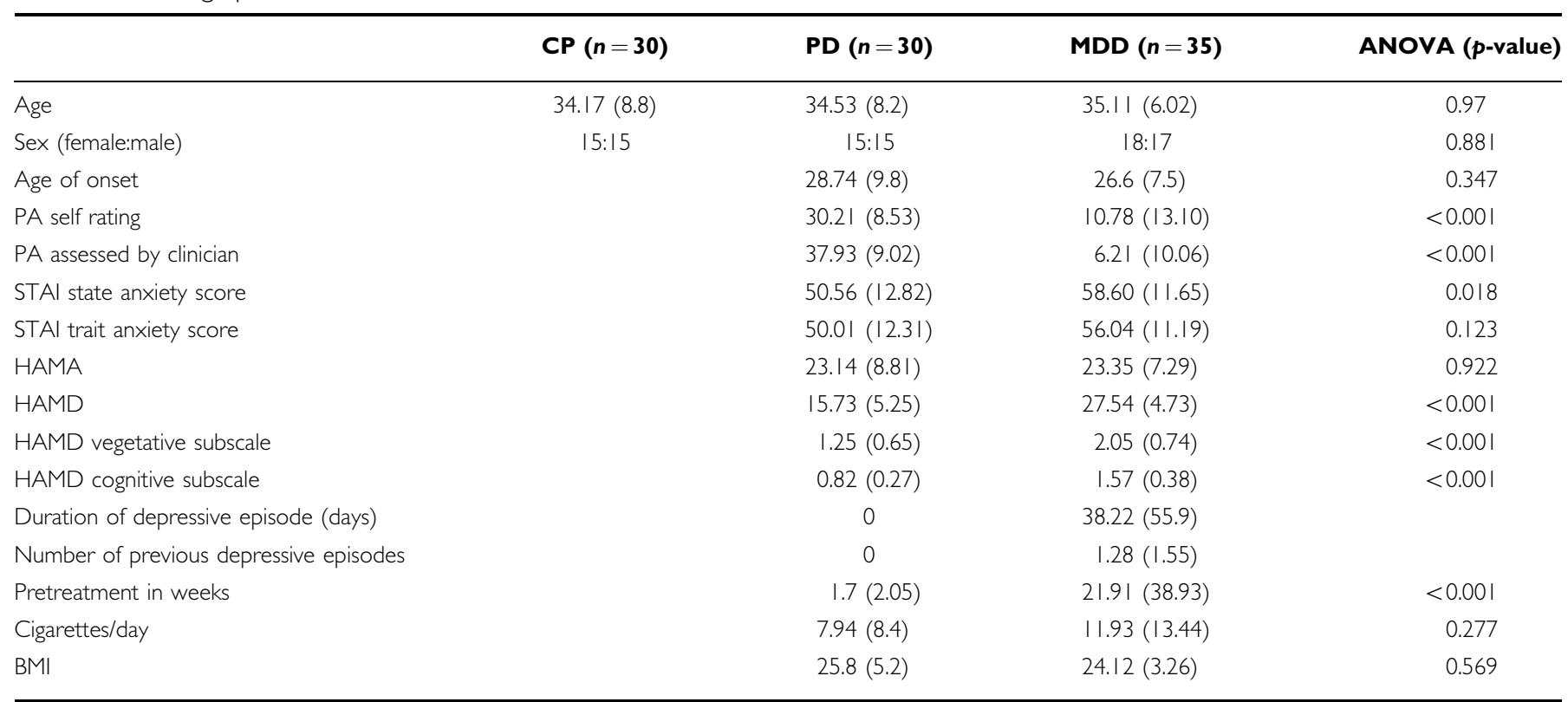

BMI: body mass index; CP: control probands; HAMA: Hamilton Anxiety Score; HAMD: Hamilton Depression Score; MDD: major depressive disorder; PA: Panic attacks and Agoraphobia Scale; PD: panic disorder; STAl: state trait anxiety inventory.

SD in brackets.

For all patients, the number of smoked cigarettes was recorded, the body weight and the height, expressed as body mass index (BMI) at the day of the Dex-CRH-Test was measured. As a previous detailed investigation failed to observe a marked influence of current antidepressant drug treatment upon Dex-CRH test outcome (Künzel et al, 2003), the test was conducted while patients were under antidepressant medication, which remained unchanged at least 1 week prior to testing. Patients with mood stabilizer or hormone therapy, such as thyroid hormone or estrogen, were not included in the study. Current benzodiazepine use or benzodiapine use during the past 2 weeks prior to the Dex-CRH-test day were exclusion criteria. In the MDD group, no electroconvulsive therapy or any other treatments apart from the stable antidepressant drug treatment were allowed during the study. For the current psychopharmacological treatment see Table 3. All patients included were Caucasian and of German origin. Turkish, Asian and Oriental ethnicity was not included to avoid population stratification.

The study has been approved from the local ethics committee of Human Experimentation of the State of Bavaria and a written informed consent was obtained from all subjects.

\section{The Dex-CRH Test}

The combined Dex-CRH test was performed as previously described in detail (Heuser et al, 1994). Briefly, patients were orally pretreated with $1.5 \mathrm{mg}$ Dex at 2300 . At 1415 the following day, the subjects were reported to the hospital of the Max-Planck-Institute. The subjects rested supine in a comfortable position and were monitored by a video camera throughout the test. Only light reading was allowed. A venous catheter was placed at 1430 and five consecutive
Table 3 Pharmacological Status

\begin{tabular}{lcc}
\hline & PD $(\boldsymbol{n}=\mathbf{3 0})$ & MDD $(\boldsymbol{n}=\mathbf{3 5})$ \\
\hline SSRI & $17(56.7 \%)$ & $13(37.1 \%)$ \\
TCA & $1(3.3 \%)$ & $1(2.9 \%)$ \\
Venlafaxine & $2(6.6 \%)$ & $1(2.9 \%)$ \\
Mirtazapine & 0 & $11(31.4 \%)$ \\
No medication & $10(33.3 \%)$ & $9(25.7 \%)$ \\
\hline
\end{tabular}

MDD: major depressive disorder; PD: panic disorder; SSRI: selective serotonine reuptake inhibitors; TCA: tricyclic antidepressant drugs.

blood samples were collected at 1500, 1530, 1545, 1600, and 1615 into tubes containing trasylol and EDTA (Bayer Inc., Germany). Blood collection was performed by a throughthe-wall technique from the adjacent room leaving the subjects undisturbed throughout the test. Between the insertion of the catheter and the first blood sample, the subjects had an accommodation time of $30 \mathrm{~min}$. A single injection of $100 \mu \mathrm{g}$ CRH (Ferring Inc., Kiel, Germany) was administered intravenously at 1502 . The reaction of the HPA system was monitored by use of the following parameters: (a) the plasma cortisol (Cbas) and ACTH (Abas) levels at 1500 before the $\mathrm{CRH}$ administration reflect solely the suppression effect of dexamethasone pretreatment; (b) the area under the curve of plasma cortisol and ACTH (Cauc, Aauc) concentration for all five time points; and (c) the area under the curve minus the baseline equivalent of cortisol and ACTH at 1500 (Cnet, Anet) reflecting the stimulating effects of $\mathrm{CRH}$ injection on HPA system. 


\section{Hormone Assays}

Hormone assays were identical to those described in Zobel et al (2001). A radioimmunoassay (RIA) kit from ICN Biomedicals, Carson, CA was used for the measurement of plasma cortisol, the detection limit was $0.3 \mathrm{ng} / \mathrm{ml}$. For plasma ACTH levels, an immunometric assay without extraction with a detection limit of $4.0 \mathrm{pg} / \mathrm{ml}$ was used (Nichols Institute, San Juan Capistrano, CA, USA).

\section{Statistical Analysis}

The three groups were matched for age, gender and ethnicity (Table 2). The area under the concentration-time course curve was computed using a trapezoidal integration (Cauc, Aauc) corrected for baseline (Cnet, Anet). Nonparametric Spearman correlations were used to investigate the influence of multiple parameters (sex, age, age of onset, number of smoked cigarettes, pharmacological/antidepressant pretreatment) and psychopathology on cortisol and ACTH levels. A multivariate analysis of variance (MANOVA) was performed to identify possible group effects. If appropriate, pair wise Games-Howell post hoc test was carried out. The Games Howell procedure maintains the experiment-wise alpha near the nominal level like the Bonferroni test but is considered superior, especially in the case of unequal variances (Jaccard et al, 1984). $\chi^{2}$ analysis and MANOVA were applied to examine differences in dexamethasone suppressor status and the interaction between Dex nonsuppression and diagnosis in MDD and PD patients, respectively. The significance level was set at $\alpha=0.05$. All statistical analyses were performed using the Statistical Package for Social Sciences (SPSS) version 12.0.

\section{RESULTS}

\section{Effects of Phenotypic Characteristics on Dex-CRH Test}

Concerning the Dex-CRH challenge test, the time course pattern of CRH-induced ACTH and cortisol secretion after Dex pretreatment was similar in all three groups, with higher increase of both parameters in the PD and MDD groups $v s$ controls (Figure 1). MANOVA was significant with $p=0.006$ (Wilk's Lambda $\mathrm{F} 8,176=2.825$ ) and univariate results showed significant group effects in the following parameters: the basal concentrations of cortisol (Cbas: $p=0.01$ ), the area under the concentration-time course curve of ACTH (Aauc: $p=0.016$ ) and cortisol (Cauc: $p=0.0003)$, the area under the concentration-time course curve corrected for baseline of ACTH (Anet: $p=0.006$ ), and Cortisol (Cnet: $p=0.003$ ). No group effect could be detected in basal concentrations of ACTH. The results of post hoc test Games-Howell are displayed in Table 4. We found a significant increase of basal cortisol levels and net area under the concentration curve of ACTH and cortisol in patients with panic attacks and depressive disorders $v s$ control patients. The area under the curve was significantly higher for ACTH and cortisol in the depression group vs controls, whereas in the panic disorder group significant differences to controls were found only for Cauc. No significant differences between the group of PD and MDD could be detected. Furthermore, no statistical difference in
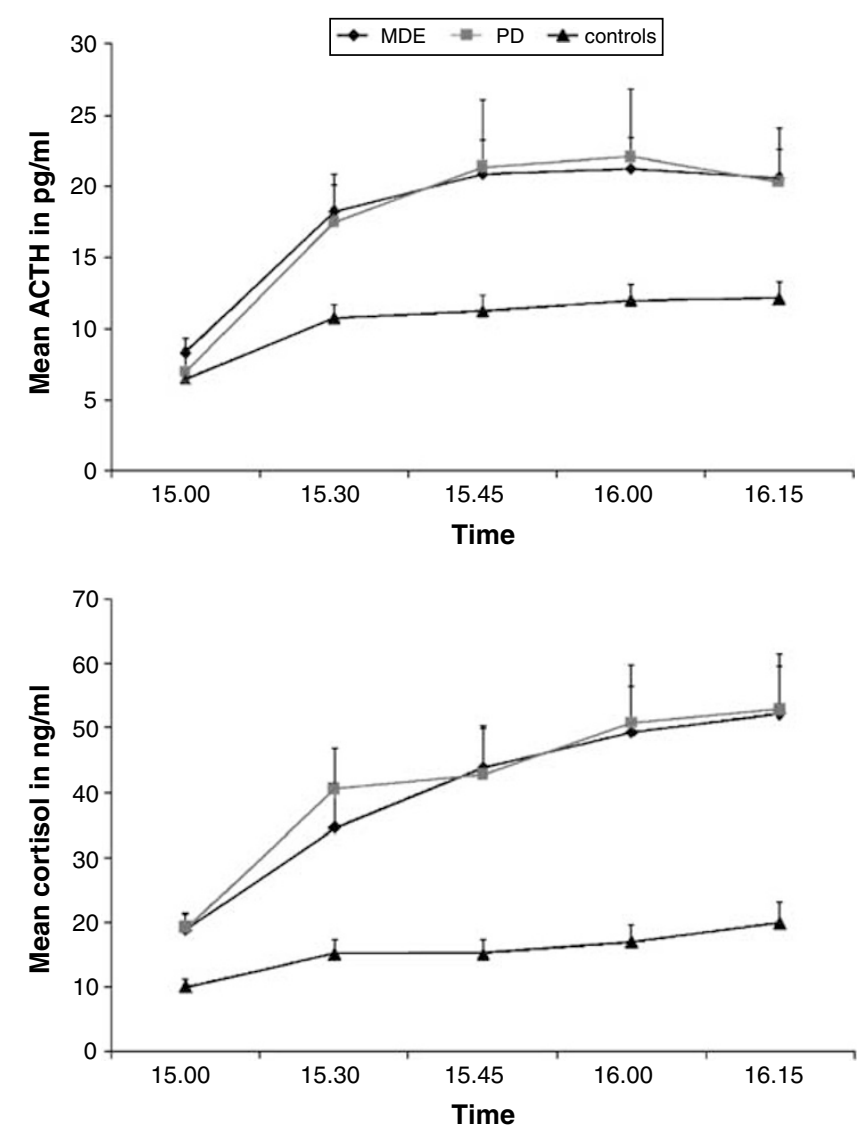

Figure I ACTH and cortisol response after dexamethasone pretreatment in controls (triangles; $n=30$ ), patients with panic disorder (PD) (rectangles; $n=30$ ) and patients with a major depressive disorder (MDD) (rhombs; $n=35$ ). Data are expressed as percentage of averaged baseline values \pm SEM

ACTH and cortisol levels could be found within the panic group in patients with and without agoraphobia.

The pharmacological treatment had no effects either on ACTH or on cortisol parameters (MANOVA $p=0.249$, Wilk's Lambda $\mathrm{F} 4,52=1.394$ ) between and within the PD and MDD groups. For panic patients with current medication (PDm) and nonmedicated patients (PDnm), the following statistical results were obtained: Cbas: PDm mean 18.61 (SD:11.0), PDnm 18.48 (8.81), $p=0.97$; Cauc: PDm 3223.58 (2369.71), PDnm 2769.46 (1527.47), $p=0.61$; Cnet: PDm 1827.32 (2245.33), PDnm 1383.37 (1179.79), $p=0.58$; Abas: PDm 7.48 (5.35), PDnm 6.69 (5.47), $p=0.72$; Aauc: PDm 1453.80 (1383.94), PDnm 1108.88 (600.79), $p=0.48$; Anet: PDm 892.09 (1131.60), PDnm 606.58 (412.08), $p=0.47$. The ANOVA results for MDD were: Cbas: MDDm mean 18.62 (SD: 13.32), MDDnm 18.87 (24.63), $p=0.97$; Cauc: MDDm 3088.85 (2313.67), MDDnm 2160.88 (2706.68), $p=0.33$; Cnet: MDDm 1691.93 (1683.69), MDDnm 745.21 (1117.67), $p=0.13$; Abas: MDDm 8.16 (5.12), MDDnm 8.78 (8.43), $p=0.79$; Aauc: MDDm 1390.50 (681.14), MDDnm 1121.26 (1048.04), $p=0.38$; Anet: MDDm 778.05 (434.37), MDDnm 462.45 (486.58), $p=0.10$.

We defined Dex suppressor status according to Cbas applying the criterion proposed by (Ising et al, 2005, 
Table 4 Basal Cortisol and ACTH Levels and in Response to CRH Administration Presented as Area Under the Curve (AUC) and Area Under the Curve Minus Cortisol and ACTH Levels at 15.00

\begin{tabular}{|c|c|c|c|c|c|c|c|}
\hline & $\mathrm{CP}(n=30)$ & PD $(n=30)$ & $\operatorname{MDD}(n=35)$ & ANOVA $F_{2,89}$ & PD vs CP p (G-H) & MDD vs CP p (G-H) & PD vs MDD p (G-H) \\
\hline Cbas & $10.09(6.25)$ & $18.57(10.16)$ & | $8.68(\mid 6.53)$ & 0.01 & 0.001 & 0.018 & 0.999 \\
\hline Cauc & I I $23.73(770.19)$ & 3077.61 (21 16.99) & $2850.23(24 \mid 4.37)$ & 0.0003 & 0.0002 & 0.001 & 0.917 \\
\hline Abas & $6.46(3.16)$ & $7.23(5.30)$ & $8.32(6.01)$ & 0.338 & & & \\
\hline Aauc & $777.94(350.37)$ & | 342.93 (| | 87.73) & | $321.27(783.47)$ & 0.016 & 0.055 & 0.02 & 0.996 \\
\hline
\end{tabular}

If appropriate, Games-Howell (G-H) post hoc tests correcting for multiple comparisons were performed. Cauc: Cbas: Cnet: CP: control probands, PD: patients with panic disorder, MDD: patients with major depressive disorder. Bold characters: $p \leqslant 0.05$.

Aauc: area under the concentration-time course curve of ACTH; Abas: basal concentrations of ACTH; the area under the concentration-time course curve corrected for baseline of ACTH; Cauc: area under the concentration-time course curve of cortisol; Cbas: basal concentrations of cortisol; Cnet: the area under the concentration-time course curve corrected for baseline of cortisol.

Table 5 Effects of Suppression versus Nonsuppression Testing Response to Dex on Cortisol Parameters in MDD and PD patients

\begin{tabular}{|c|c|c|c|c|c|c|c|}
\hline & \multicolumn{2}{|c|}{ Suppression mean (SD) } & \multicolumn{2}{|c|}{ Non-suppression mean (SD) } & \multicolumn{3}{|c|}{$p$-value univariate test results } \\
\hline & MDD; $n=28$ & PD; $n=25$ & MDD; $n=7$ & PD; $n=5$ & Diagnose & NSU & Diagnose $*$ NSU \\
\hline Cauc & $1953.66(|649.5|)$ & $2844.78(1941.47)$ & $6436.49(\mid 425.49)$ & $3700.90(2575.19)$ & NS & $<0.001$ & 0.002 \\
\hline Cnet & $1047.78(|4||.7|)$ & $1696.28(1960.83)$ & $3051.30(1327.17)$ & | 086.40 (| 897.69) & NS & 0.174 & 0.014 \\
\hline
\end{tabular}

Cauc: area under the concentration-time course curve of cortisol; Cbas: basal concentrations of cortisol; Cnet: the area under the concentration-time course curve corrected for baseline of cortisol; MDD: major depressive disorder; NS: not significant; NSU: non-suppression PD: panic disorder; SD: standard deviation. Bold characters: $p \leqslant 0.05$.

$<27.5 \mathrm{ng} / \mathrm{ml}$ ), which corresponds to the definition proposed by Heuser et al (1994). Seven of the 35 MDD and five of the $30 \mathrm{PD}$ patients were nonsuppressors while all controls were Dex suppressors $\left(\chi^{2}\right.$ test $\left.p=0.039\right)$. The number of Dex nonsuppressors did not differ between MDD and PD $(p=0.730)$. Interestingly, a significant interaction was found between Dex suppressor status (suppressor vs nonsuppressor) and diagnosis (MDD vs PD) regarding the multivariate cortisol response to the combined Dex/CRH test (interaction effect: Wilk's lambda, $p=0.004$; main effect suppressor status: $0.382, p<0.001$; main effect diagnosis: not significant). Univariate results are displayed in Table 5. PD suppressors showed larger cortisol responses to the combined Dex/CRH test than MDD suppressors, while PD nonsuppressors displayed smaller cortisol responses than MDD nonsuppressors. Additionally, we found a correlation between Cbas and Cauc in panic $(r=0.386 ; p=0.043)$ and depressed group $(r=0.804 ; p>0.001)$. Regarding Cbas and Cnet, only in the depressed group a significant positive interaction was found $(r=0.438 ; p=0.008)$.

Correlation analysis (Spearman's correlation) showed no significant effect of gender, age, BMI or the number of cigarettes smoked per day in either the MDD or panic disorder group. The age of onset of the disease was negatively correlated in the panic disorder group with Cauc $(r=-0.482 ; p=0.015)$, Cnet $(r=-0.489 ; p=0.013)$ and showed a trend for negative correlation with Aauc $(r=-0.360 ; p=0.07)$ (no correlation in the MDD group). To further investigate whether or not there is a putative interrelationship between basal plasma cortisol levels in depression and panic and the CRH response, we performed a correlation analysis between Cbas and Cnet. No correlation was found in the panic group, while a positive correlation was detected in the depression group $(r=0.438 ; p=0.008)$.

\section{Effects of Psychopathology on the Dex-CRH Test}

In the MDD group, we found a significant positive correlation between Cauc and HAMD score $(r=0.342$; $p=0.044)$. Furthermore, the cognitive HAMD subscale correlated positively with Cnet $(r=0.367 ; p=0.03)$ and Anet $(r=0.354 ; p=0.037)$. None of the anxiety scores in depressive patients (HAMA, PA self-report, PA assessed by clinicians, STAI state and trait anxiety) showed correlation effects with cortisol and ACTH parameters. In the panic disorder group, a positive correlation with HAMA (trend; $r=0.514 ; p=0.09)$, HAMD cognitive subscale $(r=0.435$; $p=0.03)$ and State Anxiety $(r=0.573 ; p=0.004)$ with Cbas was found. HAMD scores were negatively correlated $(r=-0.409 ; p=0.047)$ with Aauc.

\section{DISCUSSION}

The results of the present study demonstrate a hyperresponsiveness of the HPA system in both panic disorder and MDD patients with a similar ACTH and cortisol 
response to i.v. $\mathrm{CRH}$ administration under the suppressive action of dexamethasone. Thus, the response to the Dex$\mathrm{CRH}$ test is significantly higher in patients with panic disorder and in patients with MDD as compared to healthy controls. However, we failed to find significant differences between PD and MDD groups which have been previously shown by Schreiber et al (1996), who found that the responsiveness of the HPA system in PD patients $(n=13)$ in the Dex-CRH test was higher than in healthy controls $(n=10)$, but lower than in depressed patients $(n=10)$. The most likely explanation of this divergency regarding the failure to replicate the group difference panic disorder and major depression in our study are different inclusion criteria for the group of depressed patients. In the Schreiber et al, study, medication-free patients were examined, while most patients of our study were examined under antidepressant medication. As antidepressants exhibit their effects, at least in part, by ameliorating the resistance of glucocorticoid receptors (review: Holsboer (2000)), these drug effects are accompanied by a improvement of the HPA system dysregulation, which seems to precedes symptom resolution (Ising et al, 2005). Therefore, the depressed patients of our study are likely to display to some degree an already improved HPA system function albeit a resolution of psychopathology had not occurred at the time of the Dex-CRH test was performed. In the MD group, $31 \%$ of patients received mirtazapine therapy, which was shown by Schule et al (2003) to immediately decrease HPA system hyperdrive. In our sample, we failed to see a significant effect of mirtazapine, thus, a bias due to mirtazapine treatment is unlikely. In addition, no significant differences between medicated and nonmedicated patients in both the panic and MDD groups were found. Furthermore, in the present study, the basal cortisol levels after Dex pretreatment at 1500 were significantly higher in the panic and MDD group vs controls. Basal ACTH plasma concentrations showed no difference among the three groups. In contrast to previous studies, no gender differences could be detected in the Dex-CRH test, which is likely to be due to the smaller sample size (Heuser et al, 1994; Künzel et al, 2003).

With respect to psychopathology, a positive correlation of the HAMD score with the cortisol area under the time course curve and the net cortisol level could be found in the MDD group only. In the MDD group, scores from scales assessing anxiety were unrelated to any of the parameters of ACTH and cortisol secretion. In contrast, HAMA, state anxiety scores and the HAMD cognitive subscale were positively correlated with cortisol levels at baseline in the panic disorder group. Moreover, in the latter group, HAMD scores were even negatively correlated with the ACTH area under the curve. As patients with PD and MDD have significantly different severity of depressive symptoms, our results underline that impaired HPA regulation as evidenced by exaggerated plasma ACTH and cortisol response is not related to depressive symptoms per se, but rather indicative for a central stress-related disturbance (Holsboer, 2000; De Kloet et al, 2005). Thus, the mechanisms leading to the hyperactive response in the Dex-CRH test in panic and MDD patients could be different.

\section{HPA System Regulation in Panic Disorder and Depression}

Despite a plethora of evidence derived from preclinical studies (for review: Nemeroff, 1998; Carrasco and Van de Kar, 2003; Keck et al, 2005), it is not clear so far, whether or not HPA system-related pathways are important in the pathophysiology of anxiety disorders such as panic disorder. So far, several studies using different investigational methods failed to show robust alterations of the HPA system in patients suffering from anxiety disorders (eg Abelson and Curtis, 1996; Coryell et al, 1989; Goldstein et al, 1987). Measurements of total or free cortisol in plasma, saliva and urine have been performed in patients with different sample size, different severity of illness and at different times and periods of the day, which led to inconclusive and incomparable results.

In the present study, we found strong evidence for impaired regulation of HPA system in patients with panic disorder, underlining the well-substantiated hypothesis that this disorder could be a stress-related condition (eg Kendler et al, 2002). CRH was repeatedly shown to be an important regulator of the basal and stress-related response of the HPA system (eg Dunn and Berridge, 1990; Aguilera et al, 2001). At the behavioral level CRH displays anxiogenic effects when injected centrally (for review: Dunn and Berridge (1990)), and such effects seem to be mediated through binding of $\mathrm{CRH}$ on $\mathrm{CRH}$ receptor 1 (CRHR1) (eg Müller et al, 2003; review: Nemeroff, 1998; Keck et al, 2005). Thus, an increased interaction between CRH and its type 1 receptor, altered receptor sensitivity or enhanced signaling capacity could contribute to higher state and/or trait anxiety in individuals. Moreover, genetic predisposition, early life events and other environmental or epistatic phenomena might be of importance in the individual interaction between stressors and the activity of CRH neurocircuits (Coplan et al, 1996; Wilson and Hayward, 2005). As CRH concentrations in the cerebrospinal fluid of panic disorder patients were found to be normal (Fossey et al, 1996), there is more likely an imbalance of central CRH pathways not in general but only in distinct brain areas, which are involved in the endogenous network of fear such as the hippocampus and the amygdala (Gorman et al, 2000). Although there is a similar peripherally measurable response to $\mathrm{CRH}$ in panic and depressed patients, the central mechanisms leading to these clinical conditions might be different. In stress-related disorders, disturbances of the glucocorticoid (GR) and mineralocorticoid receptor (MR)-mediated negative feedback mechanisms, have been suggested to be peripherally reflected by aberrant neuroendocrine HPA challenge tests (Holsboer, 2000). In PD patients, in contrast to depressed patients, low rates of inadequate cortisol suppression following dexamethasone were observed (Okasha et al, 1994; Schreiber et al, 1996). In our study, $20 \%(7 / 35)$ of patients with MDD and 17\% (5/30) within the PD group fulfilled the nonsuppression criterion used here. The HAMD scores in the MDD group were significantly higher than in the PD group and comparable to the scores reported in the context of other Dex-CRH studies among depressives (Künzel et al, 2003; Kunugi et al, 2005). Thus, our finding of a low nonsuppression rate in MDD patients is likely not to be due to a lower disease severity. In patients from both 
groups, however, basal plasma cortisol concentrations were significantly elevated compared to controls, although these alterations appear less pronounced than in other studies with major depression. The pattern of cortisol response after CRH in dexamethasone pretreated suppressors and nonsuppressors differs significantly between the MDD and PD group, as the latter Dex suppressors display a higher cortisol response to $\mathrm{CRH}$ than patients with MDD. Under the condition of Dex nonsuppression, however, MDD patients show a more pronounced cortisol release than PD patients. The interpretation of this finding is limited by the small sample size of the nonsuppression group. One may speculate that the changes in HPA system regulation are of different pathophysiological relevance in both clinical conditions. In PD patients, cortisol responses to $\mathrm{CRH}$ are more similar between Dex suppressors and nonsuppressors, while the cortisol responses in MDD nonsuppressors are distinctly higher than the responses in the MDD suppressor group. The two groups of PD suppressors and nonsuppressors appear more homogenous than the MDD groups suggesting a similar pathophysiology. One possibility is that in PD Dex suppressors the sudden and intensive but temporary stress during panic attacks could lead to a reactive hyperresponsivity of the HPA system to external or internal stimuli, such as $\mathrm{CRH}$, with an overall higher cortisol release when compared to MDD patients. In an animal model of chronic social stress, epigenetic changes with modulation of BDNF gene expression were found (Tsankova et al, 2006). Similar changes have been hypothesized for the effects of stress on the glucocorticoid receptor gene (Meaney and Szyf, 2005), and could also occur in panic patients after episodes of recurrent panic attacks, which could result in a more pronounced, long lasting HPA axis overactivity and in clinical depression. Likewise, enduring stimulation of adrenal glands with ACTH could produce hyperplasia resulting in increased sensitivity to even small concentrations of ACTH. Indeed, the phenomenon of HPA system sensitization in response to repeated stressor exposure is well known from rodent experiments and has been demonstrated to be secondary to an increase in hypothalamic vasopressin (AVP) release (Dallman, 1993; Aguilera and Rabadan-Diehl, 2000; Makino et al, 2002). The differences in the suppression vs nonsuppression patterns of basal cortisol and ACTH as well as different correlations with psychopathological scales in the depression and panic groups further suggest that the neuroanatomical structures involved in the hyperactivity of HPA system in panic and depression might well be different. In panic disorder, brain structures involved in hyperarousal, perception of external and internal stimuli and cognitive judgement, could be of primary importance for HPA axis regulation. In this context, amygdala, hippocampus and prefrontal cortex would have major significance, rather than sustained CRH sensitivity and disturbances of feedback mechanisms at the hypothalamic level.

MDD nonsuppressors show the highest cortisol responses to CRH suggesting to constitute a subgroups of patients with a profound dysregulation of the HPA system, which has been described as a primary pathophysiological process in major depression (Holsboer, 2000). As MDD is perceived as a condition of chronic stress, downregulation of GR in the PVN and other brain structures such as the anterior pituitary fails to restrain hyperfunction of the HPA axis leading to nonsuppression after Dex (Makino et al, 2002). Genetic factors may contribute to these profound dysregulation and possible increased vulnerability to develop MDD (Holsboer et al, 1995). We could further speculate that the PD nonsuppression subgroup may display a crossing point between PD and MDD. Further long-term investigations are necessary to find out whether or not these patients represent a subgroup with a greater risk to develop depression in later life and to clarify whether changes in HPA system activity are causative for or secondary to panic disorder.

\section{REFERENCES}

Abelson JL, Curtis GC (1996). Hypothalamic-pituitary-adrenal axis activity in panic disorder $-24 \mathrm{~h}$ secretion of corticotropin and cortisol. Arch Gen Psychiatry 53: 323-331.

Aguilera G, Rabadan-Diehl C (2000). Vasopressinergic regulation of the hypothalamic-pituitary-adrenal axis: implications for stress adaptation. Regul Peptides 96: 23-29.

Aguilera G, Rabadan-Diehl C, Nikodemova M (2001). Regulation of pituitary corticotropin releasing hormone receptors. Peptides 22: 769-774.

Bandelow B, Broocks A, Pekrun G, George A, Meyer T, Pralle L et al (2000a). The use of the panic and agoraphobia scale (P \& A) in a controlled clinical trial. Pharmacopsychiatry 33: 174-181.

Bandelow B, Wedekind D, Sandvoss V, Broocks A, Hajak G, Pauls J et al (2000b). Diurnal variation of cortisol in panic disorder. Psychiatry Res 95: 245-250.

Brambilla F, Bellodi L, Perna G, Battaglia M, Sciuto G, Diaferia G et al (1992). Psychoimmunoendocrine aspects of panic disorder. Neuropsychobiology 26: 12-22.

Carrasco GA, Van de Kar LD (2003). Neuroendocrine pharmacology of stress. Eur J Pharmacol 463: 235-272.

Condren RM, O'Neill A, Ryan MCM, Barrett P, Thakore JH (2002). HPA axis response to a psychological stressor in generalised social phobia. Psychoneuroendocrinology 27: 693-703.

Coplan JD, Andrews MW, Rosenblum LA, Owens MJ, Friedman S, Gorman JM et al (1996). Persistent elevations of cerebrospinal fluid concentrations of corticotropin-releasing factor in adult nonhuman primates exposed to early-life stressors: Implications for the pathophysiology of mood and anxiety disorders. P Natl Acad Sci USA 93: 1619-1623.

Coplan JD, Goetz R, Klein DF, Papp LA, Fyer AJ, Liebowitz MR et al (1998). Plasma cortisol concentrations preceding lactateinduced panic-psychological, biochemical, and physiological correlates. Arch Gen Psychiatry 55: 130-136.

Coryell W, Noyes R, Schlechte J (1989). The significance of Hpa axis disturbance in panic disorder. Biol Psychiatry 25: 989-1002.

Dallman MF (1993). Stress update-adaptation of the hypothalamic pituitary-adrenal axis to chronic stress. Trends Endocrin Met 4: 62-69.

De Kloet ER, Joels M, Holsboer F (2005). Stress and the brain: from adaptation to disease. Nat Rev Neurosci 6: 463-475.

Dunn AJ, Berridge CW (1990). Physiological and behavioralresponses to corticotropin-releasing factor administration-is Crf a mediator of anxiety or stress responses. Brain Res Rev 15: $71-100$.

Engelmann M, Landgraf R, Wotjak CT (2004). The hypothalamicneurohypophysial system regulates the hypothalamic-pituitaryadrenal axis under stress: an old concept revisited. Front Neuroendocrinology 25: 132-149.

Fossey MD, Lydiard RB, Ballenger JC, Laraia MT, Bissette G, Nemeroff CB (1996). Cerebrospinal fluid corticotropin-releasing 
factor concentrations in patients with anxiety disorders and normal comparison subjects. Biol Psychiatry 39: 703-707.

Goldstein S, Halbreich U, Asnis G, Endicott J, Alvir J (1987). The hypothalamic-pituitary-adrenal system in panic disorder. $A m J$ Psychiatry 144: 1320-1323.

Goodwin RD, Lieb R, Hoefler M, Pfister H, Bittner A, Beesdo K et al (2004). Panic attack as a risk factor for severe psychopathology. Am J Psychiatry 161: 2207-2214.

Gorman JM, Kent JM, Sullivan GM, Coplan JD (2000). Neuroanatomical hypothesis of panic disorder, revised. Am J Psychiatry 157: 493-505.

Gorman JM, Coplan JD (1996). Comorbidity of depression and panic disorder. J Clin Psyhiatry 57(Suppl 10): 34-43.

Hamilton M (1959). The assessment of anxiety-states by rating. Br J Med Psychol 32: 50-55.

Hettema JM, Neale MC, Kendler KS (2001). A review and metaanalysis of the genetic epidemiology of anxiety disorders. Am J Psychiatry 158: 1568-1578.

Heuser I, Yassouridis A, Holsboer F (1994). The combined dexamethasone crh test - a refined laboratory test for psychiatric-disorders. J Psychiatry Res 28: 341-356.

Holsboer F (2000). The corticosteroid receptor hypothesis of depression. Neuropsychopharmacology 23: 477-501.

Holsboer F, Lauer CJ, Schreiber W, Krieg JC (1995). Altered hypothalamic-pituitary-adrenocortical regulation in healthysubjects at high familial risk for affective-disorders. Neuroendocrinology 62: 340-347.

Ising M, Künzel HE, Binder EB, Nickel T, Modell S, Holsboer F (2005). The combined dexamethasone/CRH test as a potential surrogate marker in depression. Prog Neuropsychopharmacol Biol Psychiatry 29: 1085-1093.

Jaccard J, Becker MA, Wood G (1984). Pairwise multiple comparison procedures - a review. Psychol Bull 96: 589-596.

Keck ME, Holsboer F (2001). Hyperactivity of CRH neuronal circuits as a target for therapeutic interventions in affective disorders. Peptides 22: 835-844.

Keck ME, Ohl F, Müller MB, Holsboer F (2005). Listening to mutant mice: a spotlight on the role of CRF/CRF receptor systems in affective disorders. Neurosci Biobehav Rev 29: 867-889.

Keck ME, Welt T, Wigger S, Renner U, Engelmann M, Holsboer F et al (2001). The anxiolytic effect of the CRH1 receptor antagonist R121919 depends on innate emotionality in rats. Eur J Neurosci 13: 373-380.

Kendler KS, Myers J, Prescott CA (2002). The etiology of phobias - an evaluation of the stress-diathesis model. Arch Gen Psychiatry 59: 242-248.

Kunugi H, Ida I, Owashi T, Kimura M, Inoue Y, Nakagawa S et al (2005). Assessment of the Dexamethasone/CRH test as a statedependent marker for hypothalamic-pituitary-adrenal (HPA) axis abnormalities in major depressive episode: a multicenter study. Neuropsychopharmacology 17: 1-9.

Künzel HE, Binder EB, Nickel T, Ising M, Fuchs B, Majer M et al (2003). Pharmacological and nonpharmacological factors influencing hypothalamic-pituitary-adrenocortical axis reactivity in acutely depressed psychiatric in-patients, measured by the Dex-CRH test. Neuropsychopharmacology 28: 21692178.

Landgraf R, Wigger A (2002). High vs low anxiety-related behavior rats: An animal model of extremes in trait anxiety. Behav Genet 32: $301-314$

Makino S, Hashimoto K, Gold PW (2002). Multiple feedback mechanisms activating corticotropin-releasing hormone system in the brain during stress. Pharmacol Biochem Behav 73: 147-158.

Meaney MJ, Szyf M (2005). Maternal care as a model for experience-dependent chromatin plasticity? Trends Neurosci 28: $456-463$.
Merikangas KR, Angst J, Eaton W, Canino G, RubioStipec M, Wacker $\mathrm{H}$ et al (1996). Comorbidity and boundaries of affective disorders with anxiety disorders and substance misuse: Results of an international task force. Br J Psychiatry 168: 58-67.

Müller MB, Zimmermann S, Sillaber I, Hagemeyer TP, Deussing JM, Timpl P et al (2003). Limbic corticotropin-releasing hormone receptor 1 mediates anxiety-related behavior and hormonal adaptation to stress. Nat Neurosci 6: 1100-1107.

Nemeroff CB (1998). The role of corticotropin-releasing factor in the pathogenesis of major depression. Pharmacopsychiatry 21: $76-82$.

Nemeroff CB, Vale WW (2005). The neurobiology of depression: Inroads to treatment and new drug discovery. J Clin Psychiatry 66(Suppl. 7): 5-13.

Okasha A, Bishry Z, Khalil AH, Darwish TA, Eldawla AS, Shohdy A (1994). Panic disorder - an overlapping or independent entity. Br J Psychiatry 164: 818-825.

Overall JE, Rhoades HM (1982). Use of the hamilton rating-scale for classification of depressive-disorders. Comp Psychiatry 23: 370-376.

Pariante CM, Thomas SA, Lovestone S, Makoff A, Kerwin RW (2004). Do antidepressants regulate how cortisol affects the brain? Psychoneuroendocrinology 29: 423-447.

Roy-Byrne PP, Stang P, Wittchen HU, Ustun B, Walters EE, Kessler RC (2000). Lifetime panic-depression comorbidity in the national comorbidity survey-association with symptoms, impairment, course and help-seeking. $\mathrm{Br} J$ Psychiatry 176: 229-235.

Schreiber W, Lauer CJ, Krumrey K, Holsboer F, Krieg JC (1996). Dysregulation of the hypothalamic-pituitary-adrenocortical system in panic disorder. Neuropsychopharmacology 15: 7-15.

Schule C, Baghai T, Zwanzger P, Ella R, Eser D, Padberg F et al (2003). Attenuation of hypothalamic-pituitary-adrenocortical hyperactivity in depressed patients by mirtazapine. Psychopharmacology 166: 271-275.

Spielberger CD (1983). Manual for the State-Trait Anxiety Inventory (STAI). Consulting Psychologists Press: PaloAlto, CA.

Stones A, Groome D, Perry D, Hucklebridge F, Evans P (1999). The effect of stress on salivary cortisol in panic disorder patients. J Affect Disord 52: 197-201.

Targum SD (1992). Cortisol response during different anxiogenic challenges in panic disorder patients. Psychoneuroendocrinology 17: 453-458.

Tsankova NM, Berton O, Renthal W, Kumar A, Neve RL, Nestler EJ (2006). Sustained hippocampal chromatin regulation in a mouse model of depression and antidepressant action. Nat Neurosci 9: 519-525.

van Gaalen MM, Stenzel-Poore MP, Holsboer F, Steckler T (2002). Effects of transgenic overproduction of $\mathrm{CRH}$ on anxiety-like behaviour. Eur J Neurosci 15: 2007-2015.

Wedekind D, Bandelow B, Broocks A, Hajak G, Ruther E (2000). Salivary, total plasma and plasma free cortisol in panic disorder. J Neural Transm 107: 831-837.

Wilson KA, Hayward C (2005). A prospective evaluation of agoraphobia and depression symptoms following panic attacks in a community sample of adolescents. J Anxiety Disord 19: 87-103.

Wittchen HU, Zaudig M, Schramm E, Spengler P, Mombour W, Klug J et al (1990). SKID. Strukturiertes Klinisches Interview für DSM-III-R. Beltz-Verlag: Weinheim, Germany.

Zobel AW, Nickel T, Kunzel HE, Ackl N, Sonntag A, Ising M et al (2000). Effects of the high-affinity corticotropin-releasing hormone receptor 1 antagonist R121919 in major depression: the first 20 patients treated. J Psychiatr Res 34: 171-181.

Zobel AW, Nickel T, Sonntag A, Uhr M, Holsboer F, Ising M (2001). Cortisol response in the combined dexamethasone/CRH test as predictor of relapse in patients with remitted depression: a prospective study. J Psychiatr Res 35: 83-94. 